\title{
A Computational Framework for Identity Based on Situation Theory
}

\author{
Janelle MASON*, Kofi KYEI *, Darrion LONG**, Hannah FOSTER***, William NICK*, James \\ MAYES***, and Albert ESTERLINE * \\ * Department of Computer Science, North Carolina A\&T State University, 1601 East Market Street, Greensboro, NC 27411 USA \\ \{jcmason, kkyei,wmnick\}@aggies.ncat.edu,esterlin@ncat.edu \\ ** Dept. of Computer Science, Technology \& Mathematics, Lincoln Univ. of Missouri, 820 Chestnut St, Jefferson City, MO 65101 USA \\ darrion.long687@my.lincolnu.edu \\ *** Department of Criminal Justice, North Carolina A\&T State University, 1601 East Market Street, Greensboro, NC 27411 USA \\ hnfoster@aggies.ncat.edu,jpmayes@ncat.edu
}

\begin{abstract}
This paper presents a computational framework for identity (initially about the culprit in a crime scene) based on Barwise's situation theory. Situations support information and can carry information about other situations. An utterance situation carries information about a described situation thanks to the constraints imposed by natural language. We are concerned with utterance situations in which identity judgments are made about the culprit in a crime scene, which is the corresponding described situation. The id-situation and crime scene along with various resource situations make up a case in the legal sense. We have developed OWL ontologies to provide concepts and principled vocabularies for encoding our scenarios in RDF, and we present an example of a SPARQL query of one of our encodings that spans situations. To follow how evidence supports hypotheses on the identity of the culprit in a crime scene, we use Dempster-Shafer theory. We tightly integrate it with our ontologies by having the representation of a case per our ontologies present a network containing situations and stitched together by objects; evidence "flows" along this network, diminishing and combining. We review the modifications of Dempster-Shafer theory required when one goes from a closed-world assumption to an open-world assumption. We review our plans regarding equational reasoning based on identities established in our id-cases, and we review the related issues regarding the meanings of URIs.
\end{abstract}

Keywords: Situation Theory, Ontologies, Dempster-Shafer theory, Identity

\section{INTRODUCTION}

The state of the art in frameworks for identity has been the SuperIdentity Project [2], which begins with some known information or element of identity, for example, a username, and transforms that element into another. For example, we use a username to determine an address. The elements are grouped according to the type of information, e.g., all email addresses, into characteristics. This creates multisets of elements of the same type. The set of all characteristics is a person's superidentity, an organized version of all known information about the individual.

An element of identity on its own, however, lacks the context needed to carry information useable as evidence. From the legal perspective, one must understand how a case is put together from various situations. Our work addresses this information and how it can be used as evidence (for the identity of an agent). We are concerned not just with the crime scene, but also with all the situations where information (e.g., fingerprints) was gathered and the processes by which its integrity is validated. For a concrete basis for information used in providing evidence, we base our framework on situation theory as developed by Barwise and his colleagues [14], the principal concern of which is how a situation supports information and carries information about other situations Our examples have been from physical crime scene investigations because our colleagues' work with biometrics, the centuries-old legal norms, and our collaboration with the Criminal Justice Program at North Carolina A\&T State University, however we are moving into the cyber world.

The topics of the remaining sections are as follows. Section 2 presents situation theory. For representing and reasoning about identity cases, we have developed OWL (Web Ontology Language) ontologies (which roughly provide schemas for data and inference); Section 3 presents these ontologies. Our approach uses Semantic Web standards (including OWL) for representing and reasoning. We encode cases in RDF (Resource Description Framework, another Semantic Web standard), using the concepts defined in our ontologies; Section 4 
presents examples of this encoding. We are interested primarily in how the evidence supports hypotheses on the identity of the culprit in a crime scene, and for this we use Dempster-Shafer theory tightly integrated with our ontologies, as explained in Section 5. Identity is an equivalence relation and supports equational reasoning. Section 6 presents our plans regarding such reasoning and discusses the meaning of URIs (Uniform Resource Identifiers, which are the primary means in the Semantic Web for "naming" individuals). Section 7 concludes and discusses future work.

\section{SITUATION THEORY}

Once we refer to or quantify over scenes modeled by first-order substructures representing visual parts of the actual world as Barwise did initially, it is natural to quantify over other substructures, i.e., over representations of other parts of the world, that is, situations. (The standard reference for situation theory is Barwise and Perry's [1]; we also refer to [3], a formalization by Devlin) According to situation semantics (situation theory applied to linguistic meaning, Barwise's main concern), the meaning of an expression is conceived as a relation between an utterance situation and a described situation. There are also resource situations to fill out references. In general, one situation can carry information about another situation by virtue of constraints. For example, this is the gist of "Smoke means fire." The constraints by which an utterance situation carries information about a described situation are the conventions of natural language.

We consider making an identity judgment (as in a crime scene investigation) to be an utterance situation (which we call an id-situation), part of a constellation of situations making up a case. The crime scene is the described situation. Resource situations include such things as where a fingerprint was filed and where one took a facial image used to train a classifier. In cases where machine learning is involved, there could be a huge number of resource situations, and we propose to use summary metadata in such cases to introduce levels of evidence.

\section{OUR ONTOLOGIES}

We utilize the Semantic Web standards to express cases in our framework. OWL is the most popular language that is used to express ontologies. Our framework consists of OWL ontologies that are used to encode information on a case in an RDF triple store. We use the query language SPARQL (SPARQL Protocol and RDF Query Language) to execute queries per each case [8], [9]. RDF expresses simple propositions as triples of the form subject property object, stating that subject and object are related by binary relation property. An example might be Fred brotherOf Ed, meaning that Fred is the brother of Ed, but in fact, the subject must be a URI or a bnode (a placeholder, like a pronoun, local to the RDF document), the property must be a URI, and the object must be a URI or a bnode or a literal.

Triples may share subject, they may share an object, and the object of one may be the subject of another. Abstractly, an RDF document presents a graph, where a triple is a directed edge from the subject to the object, labeled by the property.

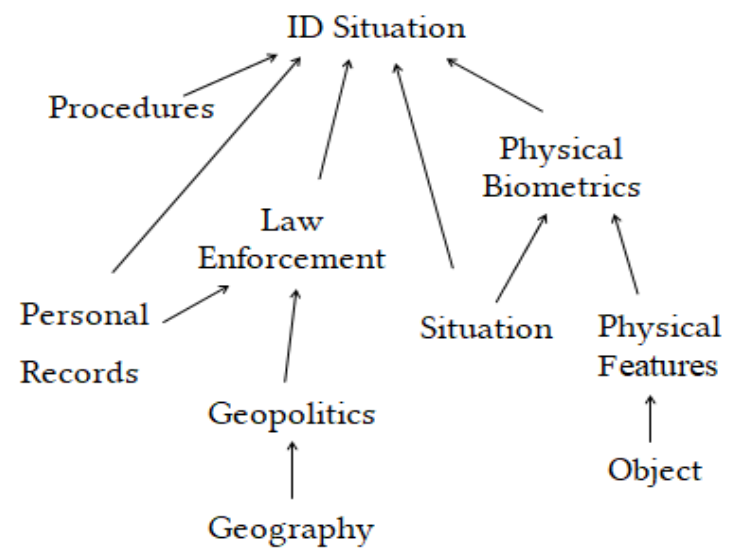

Figure 1: Framework Ontologies.

We have ontologies that are created specifically for our framework. Figure 1 depicts the relationships within our framework. Each ontology inherits from the ontology below. The Id Situation ontology is primarily focused on id-situations and constellations of situations (i.e., id-cases) that support id-situations. We assign the namespace prefix id to the Id Situation ontology. The Id Situation ontology is constructed on the Situation ontology. We assign the prefix sit to the Situation ontology. The Situation ontology contains two major classes, Situation and Infon, denoted as sit: Situation and sit: Infon, respectively. In Devlin's presentation of situation theory [15], an infon is an elementary item of information, a relation among objects at a time and place. A situation supports infons and may thereby carry information about other situations. Property sit:supports has domain sit:Situation and range sit: Infon (i.e., a situation supports an infon).

Our system of ontologies has the flexibility to include 
other ontologies that relate to the structure of a case as well as to the specific type of information (such as biometric artifacts) and procedures that are required for evidence to support id-actions (judgments of identity). We created a Physical Biometric ontology to handle biometric artifacts. The biometric artifacts are images of suspects' physical features that are registered for usage by forensic professionals. We created the Physical Features ontology to account for the information captured regarding physical biometrics. It relates specific surface features to specific persons, which lets biometric images serve as identifiers.

Our Law Enforcement ontology is the most significant of our supporting ontologies. We import the standard FOAF ontology into this ontology. At the top-level, we use the foaf:Agent class with children foaf:Organization and foaf:Person. There is an affiliation property to associate agents with organizations and a certification property to associate professionals with certificates.

\section{EXAMPLE ENCODING}

We consider two crime scenes for our examples, one takes place at a party and another occurs at a tollbooth. During the party, someone stole the family jewels from their closet. We have a list of potential suspects by reviewing the guest list. The evidence that we are able to collect from the crime scene is a group photograph from a security camera that has a guest's hand on the closet door

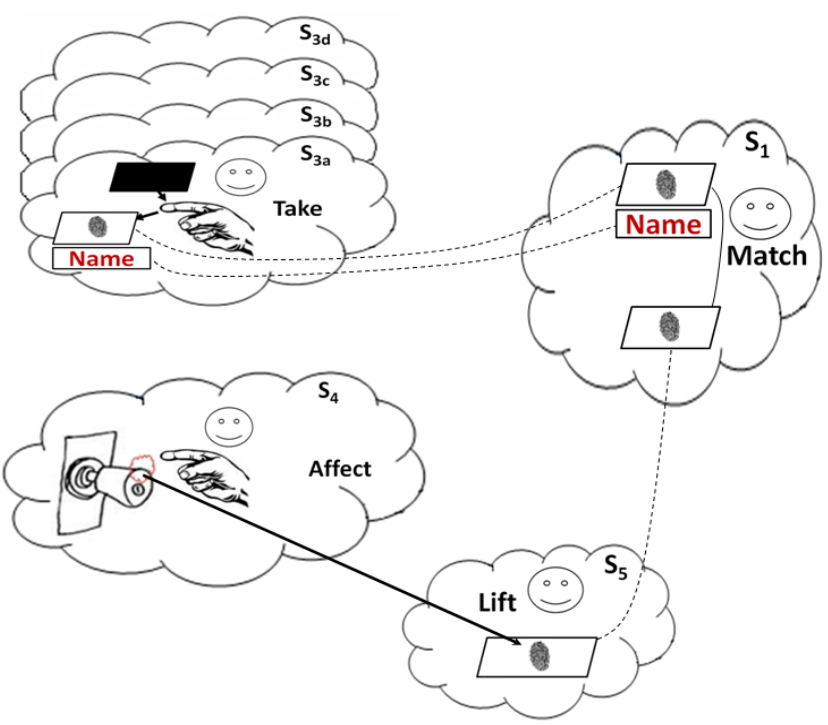

Figure 2: The fingerprint id-case.

and a fingerprint from the door knob of that door. This case targets two separate but coordinated id-situations for two pieces of evidence, the fingerprint and the snapshot. As a result, we have two coordinated id-cases.
Figure 2 depicts the fingerprint id-case with situation $\mathbf{s}_{\mathbf{1}}$ as the id-situation. This is where the forensic analyst compares fingerprints on file to the forensic evidence collected at the crime scene. For the results to be legally compelling, each fingerprint, from the police department or crime scene, must be taken and handled according to protocol in its respective situation. In each situation $\mathbf{s}_{3 \mathrm{a}}-\mathbf{s}_{\mathbf{3} \mathbf{d}}$ a suspect has their fingerprint taken and recorded on file by a forensic professional. In situation $\mathbf{s}_{\mathbf{4}}$ (part of the crime scene), the criminal touches the doorknob, which provides a forensic fingerprint. The Crime Scene Investigation team lifts the fingerprint from the doorknob in situation $\mathbf{s}_{\mathbf{5}}$ so that it can be compared against the fingerprints on file.

Figure 3 depicts the mugshot id-case with id-situation $\mathbf{s}_{2}$. In situations $\mathbf{s}_{\mathbf{6 a}}-\mathbf{S}_{\mathbf{6 d}}$, a forensic professional takes a mugshot of each suspect. Each mugshot is compared to the photo captured by the security camera in $\mathbf{s}_{7}$. In situation $\mathbf{s}_{7}$, the group is recorded by the camera, which acts as an utterance situation producing a photo as a persistent description of situation $\mathbf{s}_{\mathbf{8}}$, with the group standing near the door with someone touching the doorknob during the party. Situation $\mathbf{s}_{\mathbf{4}}$ (where a fingerprint is left by touching the doorknob) is a part of situation $\mathbf{s}_{\mathbf{8}}$, the larger scene of what occurred at that moment during the party. In this scenario, there might be sufficient evidence in each id-case on its own to identify the culprit, but perhaps the fingerprint on the doorknob is indistinct and the group photo is blurred so neither is compelling. Combining the evidence from the two cases, however, might suffice. In Section 5, we present our application of Dempster-Shafer theory, which shows how combining evidence may strengthen our case.

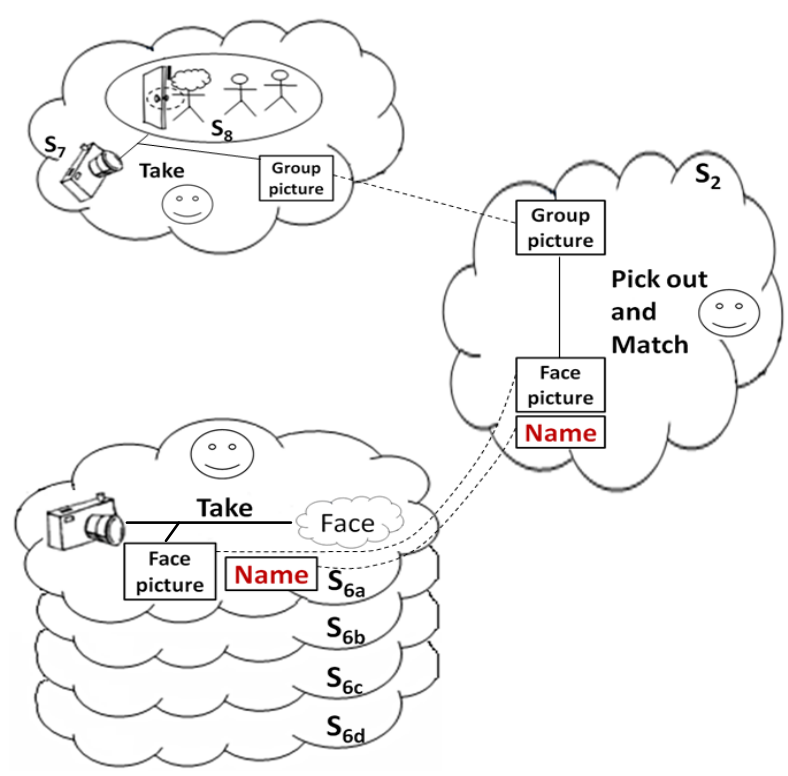

Figure 3: The mugshot id-case. 
The second crime scene takes place where a guy runs a tollbooth, depicted in Figure 4. One security camera has taken a picture of the driver's face; call this situation $\mathbf{s}_{\mathbf{f}}$.

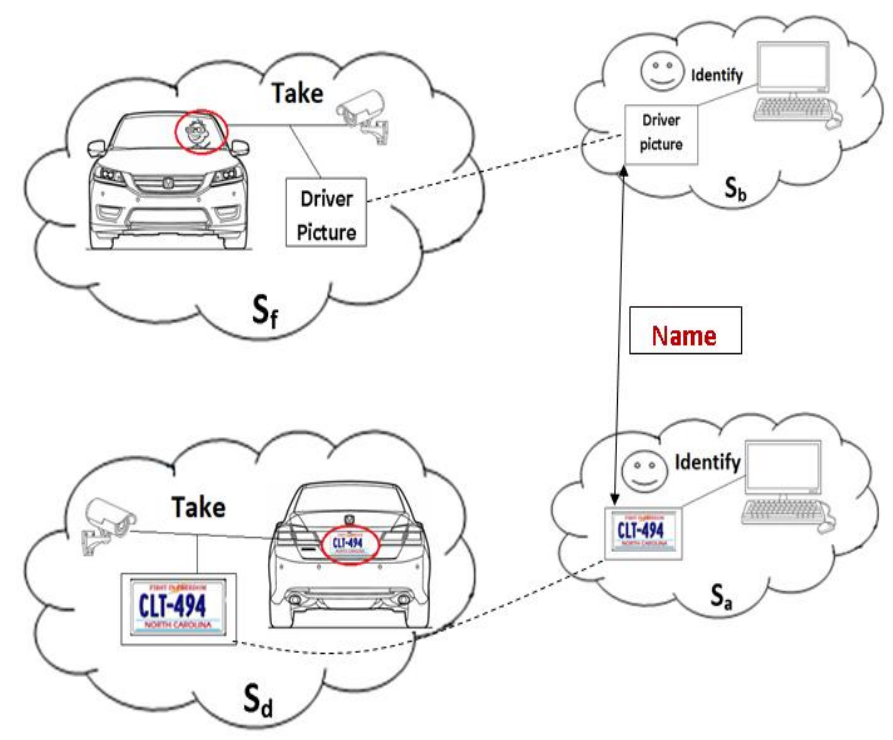

Figure 4: The tollbooth id-case.

Another camera has taken a picture of the license plate with the last digit obscure, it is clearly a North Carolina (NC) plate. Call this situation $\mathbf{s}_{\mathbf{d}}$. Both $\mathbf{s}_{\mathbf{f}}$ and $\mathbf{s}_{\mathbf{d}}$ are parts of the crime scene. They are contemporaneous but spatially disjoint.

There is an id-situation, $\mathbf{s}_{\mathbf{b}}$, where an investigator tries to match the license plate number from the photo from $\mathbf{s}_{\mathbf{d}}$ with a number on file with the NC Department of Motor Vehicles (DMV). There is probably more than one valid matching license number; there could be up to 36 . Even if there were only one, we might not have a closed case since there is no guarantee that the driver is the owner of the car. The investigation can take a multitude of turns, and many different situations might contribute. One could contact the owner and ask who was driving.

Critical situations here are where the DMV associates the license number with the owner: up to 36 such situations may be relevant. All these situations go together as the plate-matching id-case.

There is another, coordinated id-situation, $\mathbf{s}_{\mathbf{a}}$, where an attempt is made to match the photo of the driver from $\mathbf{s}_{\mathbf{f}}$ against mugshots the DMV has of all those with NC driver's licenses. This is central to another id-case, the face-matching case. This can cross over to the plate-matching case, for example, as confirmation when the owner is asked who was driving the car already identified by plate number. On its own, this case is difficult. For one thing, the DMV may not have a mugshot of the driver. Even if it has, the number of mugshots on file is so large that the chance of an unambiguous match is very small. In any case, relevant situations will include those where mugshots are taken. If the entire set of mugshots is accessed, this is a huge number of situations. In such a case, using our computational approach, rather than encoding information on the situations themselves, one would record meta-information that summarizes the set of mugshots. Even if we reduce the set of likely mugshots to a reasonable number, we still have to allow that the person is outside the set of people represented by the selected mugshots. Allowing an unbounded set of candidates is an instance of the open-world assumption, which requires special treatment when we apply Dempster-Shafer theory. We pick this up in Section 5.

\section{EVIDENCE: DEMPSTER-SHAFER THEORY}

We use Dempster-Shafer Theory (DST) [10], [5] to provide numerical values for evidence-based confidence one should have in our identities. The set of all possible elements is the frame of discernment. Mass, representing the strength of evidence, is assigned to subsets of the frame of discernment in such a way the sum of all assigned mass is 1.0. Note that mass may be assigned to singleton sets but also to non-singleton sets without being distributed over singleton components, which contrasts with the treatment of probabilities. Mass assigned to the frame of discernment itself represents ignorance. A focal element is any set assigned non-zero mass. A refinement is an analysis leading to a more detailed frame of discernment. Given a mass function, the belief function for a set that is a subset of the frame of discernment is the lower bound for likelihood, which is calculated by adding the masses of all subsets of the set, while the plausibility is the upper bound for the likelihood, which is calculated by adding the masses of the sets that overlap the set. The belief associated with a set is determined by adding the masses of all of its subsets. DST allows for the combination of multiple mass functions for different kinds of evidence to produce a new mass function for the combined evidence. There are many combination rules, appropriate for various conditions.

The structure of a case allows us to apply DST in a novel way [11], [12]. Think of the representation of a case (per our ontologies) as a network containing situations and stitched together by objects (e.g., a fingerprint is taken in one situation and used in a match in another). For DST, evidence flows through the network, diminished or combined on its way to a situation where an id-judgment 
is made. The links are constraints that allow the evidence to "flow". For example, if protocol (conventional constraints) is followed on taking the fingerprint on file, the similarity found in the id-situation can provide a certain level of evidence. We have considered combining mass functions, expanding the situation and so refining the frame of discernment, and modifying the main mass function in light of considerations such as reliability.

Zadeh showed that Demspter's rule gives counterintuitive results (Zadeh's paradox) when the two mass functions have different focal elements [7]. Smets [13] addresses such problems with his transferable belief model. He considers the frame of discernment as a set of atomic propositions rather than a set of corresponding individuals. (E.g., instead of Ed, Al, Ken, we have "It's Ed," "It's Al," and "It's Ken.") The atomic propositions are assumed disjoint. Instead of subsets of the frame of discernment, we have disjunctions of atomic propositions. Instead of a subset relation, we have implication. Smets defines three sets of propositions: $K P$ (propositions known to be possible), UP (those not considered, "unknown"), and $K I$ (those known to be impossible). The classical Dempster-Shafer and Bayesian approaches assume that $U P$ is empty; this is a closed-world assumption (CWA) the truth is necessarily in $K P$.

The open-world assumption (OWA) allows a non-empty $U P$ set (where the truth might be), and non-zero mass may be assigned to its elements. The content of the three sets gets redistributed as evidence accumulates. Proposition $A$ is transferred from $K P$ to $K I$ if the evidence shows that $A$ is impossible and from $U P$ to $K P$ if the evidence has us recall $A$ and consider it possible. Transfer from $K I$ to $K P$ or $U P$ and from $K P$ to $U P$ is not possible, and transfer from $U P$ to $K I$ has no practical effect since masses relate only to $K P$. If $\mathrm{A}$ is transferred from $K P$ to $K I$, its mass is transferred either to the propositions in $U P$ or to a more specific disjunction over the atomic propositions in $K P$. This "conditioning" forms the basis for the transferable belief model and a version of Dempster's rule that avoids Zadeh's paradox. The key notions are the OWA and that the empty subset of the frame of discernment may have non-zero mass. As mentioned, the OWA is natural for our second scenario.

Haenni [4] shows that the effect of the OWA can be achieved by adjoining to the frame of discernment an individual standing for anything else, so that the new frame is truly exhaustive. He shows that any degree of belief computed under the OWA is also obtained under the CWA and using Dempster's rule.

\section{REASONING ABOUT IDENTITY AND THE MEANING OF URIS}

We are developing software to exploit the logical nature of identity statements. We view an id-situation as involving an id-judgment, which is an assertion of an identity statement, where identity is a binary relation. The two denoting devices in an identity statement need not be verbal, as when, pointing to Fred, we say "He did it." Identity is an equivalence relation that partitions a set of denoting devices into equivalence classes; any such class provides our version of a superidentity. Asserting an identity between denoting expressions from different classes fuses the identities.

OWL provides the owl:sameAs property for identity and owl:differentFrom for its negation. OWL also supports inverse functional properties (such as the relation that makes id cards work): if $P$ is an inverse functional property and we have triples $x P z$ and $y P z$, we can infer $x=y$. As mentioned, the Semantic Web was designed with identity in mind. The non-unique name assumption (a consequence of the open-world assumption) hold that two distinct names (URIs on the Web or more recently IRIs) are not assumed to denote distinct individuals unless we are told otherwise. We are developing SWRL rules for equational reasoning (substituting identical terms) on RDF triples that use the vocabulary defined in our ontologies.

URIs are the fundamental denoting expressions ("names") on the Semantic Web, and an account of identity using Semantic-Web resources must be clear on what is required for a URI to denote something. Halpin addresses the question of how agents can determine what a URI identifies (or denotes, refers to, or means) [6]. On the pre-Semantic Web, a URI just identifies the web-page its protocol accesses, but on the Semantic Web, URIs are used to identify all resources, causing confusion between a thing and a page about it. The 'logical position' on the meaning of a URI on the Semantic Web is that it is given by whatever things satisfy the model(s) given by the formal semantics of the documents in which that URI occurs. This position is generally held by logicians, who see the Semantic Web as entirely distinct from the hypertext Web. Halpin sees this position as identical to the 'descriptivist theory of reference' popular among logic-oriented philosophers from the first half of the 20th century and according to which the referent of a name is restricted to those things that make assertions involving it true. The descriptivist theory of reference was challenged by the 'causal theory of reference,' according to which an 
agent fixes a name to a referent known through direct acquaintance, and a causal chain to a current user of the name from past users allows its referent to be known across time. This philosophical position (according to Halpin) is the same as the 'direct reference position' on the meaning of a URI, that it is whatever was intended by the owner, a viewpoint generally held by many Web architects, who see no difference here between the Semantic Web and the entire Web. The referent of a URI is established by fiat by the owner and then communicated to others in a causal chain by creating web pages and Semantic-Web statements containing the URI. Halpin states that both positions ignore the notion of 'sense,' something that is objective in being a shared part of a public language. He thus presents a third position, the 'public language position,' where he grounds the notion of sense in Wittgenstein's analysis of "meaning as use" in a community. Here what a URI means is "the set of search terms that leads multiple users to discover the URI in the context of satisfying a particular information need."

URIs in our RDF encodings are clearly covered by the direct reference position as legal professionals have the task of assigning URIs to suspects, equipment, fingerprints, and so on. But there is still some aspect of the public language position since, in assigning URIs, the professionals follow conventions common to their profession. And there is also some aspect of the logical position since certain logical rules must be followed if the URIs will actually denote things: e.g., the same URI cannot be assigned twice, and certain type restrictions must be obeyed. The logical position is behind the development of ontologies (where, however, most of the terms are for universals). Still, as most ontologies formalize concepts and terms already used in their domains, there is a strong aspect of the public language position in forming ontologies.

\section{CONCLUSION}

We present a computational framework for identity based on Barwise's situation theory. Situations support information and can carry information about other situations. An utterance situation carries information about a described situation thanks to the constraints imposed by natural language. We are concerned with utterance situations in which identity judgments are made about the culprit in a crime scene, which is the corresponding described situation. The id-situation and crime scene along with various resource situations make up an id-case. In an investigation, there may be several coordinated id-cases that use different means in search of the same judgment. We have developed OWL ontologies to provide concepts for encoding our scenarios in RDF.

To follow how evidence supports hypotheses on the identity of the culprit, we use Dempster-Shafer theory tightly integrated with our ontologies. We focused on cases where the set of candidates is unbounded and reviewed the modifications of Dempster-Shafer theory required when one goes to an open-world assumption (OWA). We have the challenge of encoding OWA scenarios for our framework. The scenarios will contain OWA for Dempster-Shafer theory, OWL, and partiality with situation theory. In regards to Dempster-Shafer theory, we will develop the proper rules to determine how to handle evidence in the OWA. For OWL, we will determine how to represent the structure of OWA in a legal case.

As mentioned, the causal theory of reference fits quite naturally into our approach. In fact, we generalize this causal theory beyond linguistic reference. In a legal setting, Chain of Custody theory addresses what is needed "to ensure the integrity of evidence" all along the chain of those possessing the evidence and perhaps passing it along [17]. A chain of custody involves a sequence of what we call information-relevant actions. Barwise recognized one kind of information-relevant action, utterances (of declarative statements), but we recognize a multitude of kinds, for example, taking and duplicating a fingerprint. While a legal perspective emphasizes what authority was in control, a more general perspective adds the existence of a causal chain from the physical feature in question to where the information is used. (See [16] for further discussion of this point.) As also mentioned, there is some aspect of the logical position on the meanings of URIs as certain logical rules must be followed. The logical position also extends to the broader realm, beyond linguistic reference, since, for information to count as evidence, the physical processes making up the causal chain must occur in a setting where conventions are followed. Note that the links in the causal chain and in the chain of custody are where, in the application of Dempster-Shafer theory, mass may be discounted (for example, because the professional taking the fingerprint on file had not renewed her certification recently). Future work will include working out principled ways to represent causal chains and chains of custody to allow mass functions to be modified in consistent ways.

A major challenge for equational reasoning is the existence of so-called referentially opaque contexts [18], where substituting identicals does not preserve the truth of 
a statement. The scope of a verb of propositional attitude (e.g., "believe") is typically referentially opaque. For example, suppose that the statement "John believes that Jupiter is the fourth planet from the sun" is true, and "Jupiter is the fifth planet from the sun" is true. From the truth of these two statements, it does not follow, however, that "John believes that the fifth planet from the sun is the fourth planet from the sun." Other contexts that are typically referentially opaque include modal contexts (within the scope of, e.g., "It is necessary that ..."). Whenever we have access to a set of statements from another source (say, on the Web), we can ask whether these statements are within a referentially opaque context. We need a principled way to address this. We also reviewed the related issues regarding the meanings of URIs.

\section{ACKNOWLEDGMENTS}

This research is based upon work supported by the U.S. Government including the National Science Foundation. The views and conclusions contained herein are those of the authors and should not be interpreted as necessarily representing the official policies or endorsements, either expressed or implied, of the U.S. Government. The U.S. Government is authorized to reproduce and distribute reprints for Governmental purposes notwithstanding any copyright annotation thereon.

\section{REFFERENCES}

[1] Jon Barwise and John Perry. 1998. Situations and Attitudes (new ed.). Center for the Study of Language and Information, New York, NY.

[2] S. Creese D. Hodges and M. Goldsmith. 2012. A model for identity in the cyber and natural universes. In European Intelligence and Security Informatics Conf. (EISIC). 115-122.

[3] Keith Devlin. 1995. Logic and Information. Cambridge University Press.

[4] Rolf Haenni. 2006. Uncover Dempster's rule where it is hidden. In Information Fusion, 2006 9th International Conference on. IEEE, 1-8.

[5] Joseph Y Halpern. 2005. Reasoning about uncertainty. MIT press.

[6] Harry Halpin. 2011. Sense and Reference on the Web. Minds \& Machines 21 (2011), 153-178.

[7] Zadeh L. (Ed.). [n. d.]. A mathematical theory of evidence (book review). ([n. d.]).

[8] M. McDaniel, E. Sloan, S. Day, J. Mayes, W. Nick, K. Roy, and A. Esterline. 2017. Situation-Based Ontologies for a Computational Framework for
Identity Focusing on Crime Scenes. In Proc. IEEE Conf. on Cognitive \& Computational Aspects of Situation Management (CogSIMA '17). Savanna, GA.

[9] M. McDaniel, E. Sloan, W. Nick, J. Mayes, and A. Esterline. 2017. Ontologies for Situation-Based Crime Scene Identities. In Proc. IEEE SoutheastCon. Charlotte, NC.

[10] Glenn Shafer. 1976. A mathematical theory of evidence. Vol. 42. Princeton University Press.

[11] E. Sloan, M. McDaniel, W. Nick, J. Mayes, and A. Esterline. 2016. Structure and Evidence in Identity Cases. In Proc. IEEE SoutheastCon (IEEE Symp. Series on Computational Intelligence (IEEE SSCI 2016)).

[12] E. Sloan, M. McDaniel, W. Nick, J. Mayes, and A. Esterline. 2017. Marshalling Situation-based Evidence in Identity Cases. In Proc. IEEE SoutheastCon. Charlotte, NC.

[13] Ph Smets. 1993. An axiomatic justifiaction for the use of belief function to quantify beliefs. In IJCAI, Vol. 93. 598-603.

[14] J. Barwise and J. Perry, Situations and attitudes, Reissued. Stanford, CA: CSLI Pubs., 1999.

[15] Devlin Keith. Logic and Information. Cambridge University Press. 1995.

[16] A. Esterline, W. Nick, and J. Mason, "Situations, Information, and Evidence," accepted for the 2018 IEEE Conference on Cognitive and Computational Aspects of Situation Management (CogSIMA).

[17] P. C. Giannelli, "Chain of custody," School of Law, Case Western Reserve University, Report, 1993. [Online]. Available: http://scholarlycommons.law.case.edu/faculty_public ations $/ 309$.

[18] W. V. O. Quine, Word and Object, the MIT Press, 1960. 